\title{
HOMOLOGICAL DIMENSION OF SMASH PRODUCT OVER QUASITRIANGULAR WEAK HOPF ALGEBRA
}

\author{
Zhong-wei Wang \\ Received: 29 May 2014; Revised: 30 November 2014 \\ Communicated by Abdullah Harmancı
}

\begin{abstract}
Let $(H, R)$ be a quasitriangular weak Hopf algebra, and $A$ a quantum commutative weak $H$-module algebra. We establish the relationship of homological dimensions between weak smash product algebra $A \# H$ and $A$ under some conditions. As an application, we consider the case of twisted weak Hopf algebra.
\end{abstract}

Mathematics Subject Classification (2010): $16 \mathrm{~T} 05$

Keywords: Quasitriangular weak Hopf algebra, quantum commutative weak module algebra, weak smash product, homological dimension

\section{Introduction}

Weak Hopf algebras were introduced by Böhm et al. in [3], as a generalized notation of Hopf algebras and groupoid algebras. Roughly speaking, a weak Hopf algebra is an object which has both algebra and coalgebra structures with some relations between them and that possesses an antipode which does not necessarily satisfy the usual convolution equalities with the identity morphism.

The main difference between ordinary and weak Hopf algebras is that the comultiplication of the latter is no longer required to preserve the unit, or equivalently, the counit is not required to be an algebra homomorphism. The motivations to study weak Hopf algebras mainly come from their connection with the theory of algebra extensions, the applications in the study of dynamical twists of Hopf algebras and their link with quantum field theories and operator algebras (see [13]).

The notion of quasitriangular weak Hopf algebras was introduced by Nikshych and Vainerman in [13] and consequently studied in [12], where it was shown that quasitriangular weak Hopf algebras play an important role in the quantum group theory, particularly in knot theory. It is known that the $R$-matrix in a quasitriangular weak Hopf algebra can generate a solution for the quantum Yang-Baxter

This work is supported by the Scientific Research Foundations of Jinling Institute of Technology: jit-b-201402 and 2014-jit-n-08, respectively. 
equation $R^{12} R^{13} R^{23}=R^{23} R^{13} R^{12}$ which frequently appears in many contexts of mathematical physics. From categorical point of view quasitriangular Hopf algebras are characterized by the fact that its representation categories (categories of modules having finite dimensional over ground field) are braided rigid tensor categories.

The relationship of homological dimensions between an algebra and its some subalgebra have been investigated in many papers up to now. For example, Auslander proved in [1] that gl.dim $(\Lambda G)=g l \cdot \operatorname{dim}(\Lambda)$ for a skew group algebra $\Lambda G$ with the order of $G$ invertible in $\Lambda$. In [20] Yang established the relationship of homological dimension between smash product algebra $A \# H$ and quantum commutative $H$-module algebra $A$ over a Hopf algebra $H$. It was shown that $g l \cdot \operatorname{dim}(A \# H)=n$ if and only if $\operatorname{gl} \cdot \operatorname{dim}(A)=n$ and there exists an element $c \in A$ such that $t \cdot c=1_{A}$, where $t \in H$ is a left integral. Jia and Li proved that for a quantum commutative weak $H$-module algebra $A$ over a semisimple quasitriangular weak Hopf algebra $H$, the global dimension of weak smash product $A \# H$ equals that of $A$ in [8]. Let $H$ be a semisimple weak Hopf algebra, $A$ a weak $H$-comodule algebra and $B$ the coinvariant subalgebra of $A$. If the algebra extension $A / B$ is $H$-Galois, then it was proved by Zhou in [22] the global dimension of $A$ is no more than that of $B$.

Inspired by [20], in this paper we shall investigate the relationship of homological dimensions between a quantum commutative weak $H$-module algebra $A$ over a quasitriangular weak Hopf algebra $H$ and that of weak smash product $A \# H$. As a main result of Section 2, we show that $g l \cdot d i m(A \# H)=n$ if and only if $g l \cdot \operatorname{dim}(A)=$ $n$ and there exists an element $c \in A$ such that $t \cdot c=1_{A}$, where $t \in H$ is a left integral. As an application, we consider the case of twisted weak Hopf algebra in Section 3 .

Throughout this paper, we always work over a fixed field $k$. Any unexplained definitions and notations of algebras, coalgebras, modules and comodules may be found in [10] or [16].

Definition 1.1. [3] Let $H$ be both an algebra and a coalgebra. Then $H$ is called a weak bialgebra if it satisfies the following conditions:

$$
\begin{aligned}
\Delta(x y) & =\Delta(x) \Delta(y) \\
\varepsilon(x y z) & =\Sigma \varepsilon\left(x y_{1}\right) \varepsilon\left(y_{2} z\right)=\Sigma \varepsilon\left(x y_{2}\right) \varepsilon\left(y_{1} z\right), \\
\Delta^{2}\left(1_{H}\right) & =\left(\Delta\left(1_{H}\right) \otimes 1_{H}\right)\left(1_{H} \otimes \Delta\left(1_{H}\right)\right) \\
& =\left(1_{H} \otimes \Delta\left(1_{H}\right)\right)\left(\Delta\left(1_{H}\right) \otimes 1_{H}\right),
\end{aligned}
$$

for any $x, y, z \in H$, where $\Delta\left(1_{H}\right)=1_{1} \otimes 1_{2}$ and $\Delta^{2}=\left(\Delta \otimes i d_{H}\right) \circ \Delta$. 
Moreover, if there exists a $k$-linear map $S: H \rightarrow H$, called antipode, satisfying the following axioms for all $h \in H$ :

$$
\Sigma h_{1} S\left(h_{2}\right)=\Sigma \varepsilon\left(1_{1} h\right) 1_{2}, \quad \Sigma S\left(h_{1}\right) h_{2}=\Sigma \varepsilon\left(h 1_{1}\right) 1_{2}, \quad \Sigma S\left(h_{1}\right) h_{2} S\left(h_{1}\right)=S(h),
$$

then the weak bialgebra $H$ is called a weak Hopf algebra.

Definition 1.2. [12] A quasitriangular weak Hopf algebra is a pair $(H, R)$, where $H$ is a weak Hopf algebra and $R=R^{(1)} \otimes R^{(2)} \in \Delta^{o p}(1)(H \otimes H) \Delta(1)$ satisfies the following conditions:

$$
\begin{aligned}
\Delta^{o p}(h) R & =R \Delta(h) \\
\Sigma \Delta\left(R^{(1)}\right) \otimes R^{(2)} & =\Sigma R^{(1)} \otimes r^{(1)} \otimes R^{(2)} r^{(2)} \\
\Sigma R^{(1)} \otimes \Delta\left(R^{(2)}\right) & =\Sigma R^{(1)} r^{(1)} \otimes r^{(2)} \otimes R^{(2)}
\end{aligned}
$$

for all $h \in H$, and such that there exists $R^{-1} \in \Delta(1)(H \otimes H) \Delta^{o p}(1)$ with

$$
R R^{-1}=\Delta^{o p}(1), R^{-1} R=\Delta(1)
$$

where $R=\Sigma R^{(1)} \otimes R^{(2)}=\Sigma r^{(1)} \otimes r^{(2)}, \Delta^{o p}(h)=\Sigma h_{2} \otimes h_{1}$ for all $h \in H$.

For any weak bialgebra $H$, it is well known that the maps $\Pi^{L}, \Pi^{R}, \bar{\Pi}^{L}$ and $\bar{\Pi}^{R}: H \rightarrow H$ are projections. They are given by the formulas: $\Pi^{L}(h)=\Sigma \varepsilon\left(1_{1} h\right) 1_{2}$, $\bar{\Pi}^{L}(h)=\Sigma \varepsilon\left(1_{2} h\right) 1_{1}, \Pi^{R}(h)=\Sigma \varepsilon\left(h 1_{2}\right) 1_{1}$ and $\bar{\Pi}^{R}(h)=\Sigma \varepsilon\left(h 1_{1}\right) 1_{2}$. Denote the image of the map $\Pi^{L}$ by $H^{L}$ and the image of the map $\Pi^{R}$ by $H^{R}$. Then we also have $H^{L}=\operatorname{Im} \bar{\Pi}^{R}$ and $H^{R}=\operatorname{Im} \bar{\Pi}^{L}$. Let $t \in H$. Then $t$ is said to be a left integral, if $h t=\Pi^{L}(h) t$ for all $h \in H$.

Definition 1.3. [11] Let $H$ be a weak bialgebra. An algebra $A$ is called a weak left $H$-module algebra if $A$ is a left $H$-module via $h \otimes a \mapsto h \cdot a$ such that

$$
h \cdot(a b)=\Sigma\left(h_{1} \cdot a\right)\left(h_{2} \cdot b\right), h \cdot 1_{A}=\Pi^{L}(h) \cdot 1_{A},
$$

for all $h \in H, a, b \in A$.

Definition 1.4. [11] Let $H$ be a weak Hopf algebra with bijective antipode $S$ and $A$ a weak left $H$-module algebra. The smash product algebra $A \# H$ of $A$ with $H$ is defined on the vector space $A \otimes_{H^{L}} H$, where $A$ is a right $H^{L}$-module via $a \cdot z=S^{-1}(z) \cdot a=a\left(z \cdot 1_{A}\right)$, its multiplication is given by

$$
(a \# h)(b \# g)=\Sigma a\left(h_{1} \cdot b\right) \# h_{2} g
$$

for all $a \in A, z \in H^{L}$, and the unit of $A \# H$ is $1_{A} \# 1_{H}$. 
By [8], we know that $M$ is a left $A \# H$-module via $(a \# h) \cdot m=a \cdot(h \cdot m)$ if and only if $M$ is a left $A$-module and a left $H$-module such that

$$
h \cdot(a \cdot m)=\Sigma\left(h_{1} \cdot a\right) \cdot\left(h_{2} \cdot m\right) .
$$

for all $h \in H, a \in A, m \in M$. Hence, the weak left $H$-module algebra $A$ is particularly a left $A \# H$-module via $(a \# h) \cdot b=a(h \cdot b)$ for all $h \in H, a, b \in A$.

Definition 1.5. [8] Let $(H, R)$ be a quasitriangular weak Hopf algebra, and $A$ a weak left $H$-module algebra. We say that $A$ is quantum commutative with respect to $(H, R)$ if

$$
a b=\Sigma\left(R^{(2)} \cdot b\right)\left(R^{(1)} \cdot a\right)
$$

for all $a, b \in A$.

If $A$ is quantum commutative with respect to $(H, R)$, then for all $a, b \in A$,

$$
a b=\Sigma\left(R^{(1)} \cdot b\right)\left(S^{-1}\left(R^{(2)}\right) \cdot a\right) .
$$

In fact, since $\left(i d \otimes S^{-1}\right)(R)=R^{-1}$ by [12], we have

$$
\begin{aligned}
\Sigma\left(R^{(1)} \cdot b\right)\left(S^{-1}\left(R^{(2)}\right) \cdot a\right) & =\Sigma\left(R^{(2)} S^{-1}\left(r^{(2)}\right) \cdot a\right)\left(R^{(1)} r^{(1)} \cdot b\right) \\
& \stackrel{(9)}{=} \Sigma\left(1_{1} \cdot a\right)\left(1_{2} \cdot b\right)=a b .
\end{aligned}
$$

Definition 1.6. [4] Let $H$ be a weak bialgebra, and $B$ a right $H$-comodule, which is also an algebra with a unit, such that

$$
\begin{aligned}
\rho(a b) & =\rho(a) \rho(b), \\
\Sigma a_{(0)} \otimes \Pi^{L}\left(a_{(1)}\right) & =\Sigma a 1_{(0)} \otimes 1_{(1)},
\end{aligned}
$$

for all $a, b \in B$. Then $B$ is called a weak right $H$-comodule algebra.

Let $H$ be a weak Hopf algebra, and $B$ a weak right $H$-comodule algebra. We define the subalgebra of coinvariants as $C:=B^{c o H}=\left\{x \in B \mid \Sigma x_{(0)} \otimes x_{(1)}=\right.$ $\left.\Sigma x_{(0)} \otimes \Pi^{L}\left(x_{(1)}\right)\right\}$. By [15], we know that $C=\left\{x \in B \mid \Sigma x_{(0)} \otimes x_{(1)}=\Sigma x 1_{(0)} \otimes\right.$ $\left.1_{(1)}\right\}=\left\{x \in B \mid \Sigma x_{(0)} \otimes x_{(1)}=\Sigma 1_{(0)} x \otimes 1_{(1)}\right\}$.

If the following canonical map

$$
\text { can }: B \otimes_{C} B \rightarrow B \otimes_{H^{R}} H, \operatorname{can}(a \otimes b)=\Sigma a b_{(0)} \otimes b_{(1)},
$$

is bijective, then the extension $B / C$ is called weak right $H$-Galois, where $B$ is a right $H^{R}$-module via $a \cdot y=\Sigma \varepsilon\left(a_{(1)} y\right) a_{(0)}$, and $H$ a left $H^{R}$-module via $y \cdot h=h S^{-1}(y)$, for any $a \in B, h \in H, y \in H^{R}$.

Define

$$
c a n^{\prime}: B \otimes_{C} B \rightarrow B \otimes_{H^{R}} H, \operatorname{can}^{\prime}(a \otimes b)=\Sigma a_{(0)} b \otimes a_{(1)},
$$


and

$$
\psi: B \otimes_{H^{R}} H \rightarrow B \otimes_{H^{R}} H, \psi(a \otimes h)=\Sigma a_{(0)} \otimes a_{(1)} S(h)
$$

for all $a \in B, h \in H$. Then $\operatorname{can}^{\prime}=\psi \circ$ can.

As a matter of fact, we have

$$
\Sigma 1_{(0)} \otimes_{H^{R}} 1_{(1)}=1_{B} \otimes_{H^{R}} 1_{H},
$$

since

$$
\begin{aligned}
\Sigma 1_{(0)} \otimes_{H^{R}} 1_{(1)} & =\Sigma 1_{(0)} \otimes S\left(1_{(1)}\right) \cdot 1_{H}=\Sigma 1_{(0)} \cdot S\left(1_{(1)}\right) \otimes 1_{H} \\
& =\Sigma \varepsilon\left(1_{(1)} S\left(1_{(2)}\right)\right) 1_{(0)} \otimes 1_{H}=\Sigma \varepsilon\left(\Pi^{L}\left(1_{(1)}\right)\right) 1_{(0)} \otimes 1_{H} \\
& =1_{B} \otimes_{H^{R}} 1_{H} .
\end{aligned}
$$

Then for any $a, b \in B$, we have

$$
\begin{aligned}
\psi \circ \operatorname{can}(a \otimes b) & =\psi\left(\Sigma a b_{(0)} \otimes b_{(1)}\right)=\Sigma a_{(0)} b_{(0)} \otimes a_{(1)} b_{(1)} S\left(b_{(2)}\right) \\
= & \Sigma a_{(0)} b_{(0)} \otimes a_{(1)} \Pi^{L}\left(b_{(1)}\right) \stackrel{(16)}{=} \Sigma a_{(0)} b 1_{(0)} \otimes a_{(1)} 1_{(1)} \\
\stackrel{(18)}{=} & \Sigma a_{(0)} b \otimes a_{(1)},
\end{aligned}
$$

as required.

\section{Homological dimensions over quasitriangular weak Hopf algebra}

In this section, we always assume that $(H, R)$ is a finite dimension quasitriangular weak Hopf algebra. Then the antipode $S$ of $H$ is bijective by [3], and $H$ has a dual pair of left integrals $(t, \lambda)$ by Theorem 4.1 in [17]. Let $A$ be a quantum commutative weak left $H$-module algebra, and $A \# H$ the weak smash product.

Lemma 2.1. Let $M$ is left $A \# H$-module. If there is a trace one element and $M$ is projective as an $A$-module, then $M$ is also projective as an A\#H-module.

Proof. Let $M, N$ be left $A \# H$-modules, and $\omega: M \rightarrow N$ be an $A$-module morphism. Denote the trace one element by $c$. Define the map $\widehat{\omega}: M \rightarrow N$ by

$$
\widehat{\omega}(m)=\Sigma t_{2} \cdot\left[\left(R^{(1)} \cdot c\right) \cdot \omega\left(S^{-1}\left(t_{1} R^{(2)}\right) \cdot m\right)\right]
$$

for all $a \in A$ and $m \in M$. Then, $\widehat{\omega}$ is an $A \# H$-module morphism.

For any $a \in A, h \in H$ and $m \in M$, on one hand, since $\Sigma S^{-1}\left(h_{2}\right) h_{1}=\bar{\Pi}^{R}(h)$ and

$$
\Sigma h_{1} \otimes \Pi^{L}\left(h_{2}\right)=\Sigma 1_{1} h \otimes 1_{2}, \Sigma \bar{\Pi}^{R}\left(h_{1}\right) \otimes h_{2}=\Sigma 1_{2} \otimes h S\left(1_{1}\right),
$$


for $h \in H$ by [18], we have

$$
\begin{aligned}
& h \cdot \widehat{\omega}(m)=\Sigma h t_{2} \cdot\left[\left(R^{(1)} \cdot c\right) \cdot \omega\left(S^{-1}\left(t_{1} R^{(2)}\right) \cdot m\right)\right] \\
& =\Sigma h 1_{2} t_{2} \cdot\left[\left(R^{(1)} \cdot c\right) \cdot \omega\left(S^{-1}\left(1_{1} t_{1} R^{(2)}\right) \cdot m\right)\right] \\
& =\Sigma h 1_{2} t_{2} \cdot\left[\left(R^{(1)} \cdot c\right) \cdot \omega\left(S^{-1}\left(t_{1} R^{(2)}\right) S^{-1}\left(1_{1}\right) \cdot m\right)\right] \\
& =\Sigma h S\left(1_{1}\right) t_{2} \cdot\left[\left(R^{(1)} \cdot c\right) \cdot \omega\left(S^{-1}\left(t_{1} R^{(2)}\right) 1_{2} \cdot m\right)\right] \\
& \stackrel{(20)}{=} \quad \Sigma h_{2} t_{2} \cdot\left[\left(R^{(1)} \cdot c\right) \cdot \omega\left(S^{-1}\left(t_{1} R^{(2)}\right) \bar{\Pi}^{R}\left(h_{1}\right) \cdot m\right)\right] \\
& =\Sigma h_{3} t_{2} \cdot\left[\left(R^{(1)} \cdot c\right) \cdot \omega\left(S^{-1}\left(h_{2} t_{1} R^{(2)}\right) h_{1} \cdot m\right)\right] \\
& =\Sigma\left(h_{2} t\right)_{2} \cdot\left[\left(R^{(1)} \cdot c\right) \cdot \omega\left(S^{-1}\left(\left(h_{2} t\right)_{1} R^{(2)}\right) h_{1} \cdot m\right)\right] \\
& =\Sigma \Pi^{L}\left(h_{2}\right)_{2} t_{2} \cdot\left[\left(R^{(1)} \cdot c\right) \cdot \omega\left(S^{-1}\left(\Pi^{L}\left(h_{2}\right)_{1} t_{1} R^{(2)}\right) h_{1} \cdot m\right)\right] \\
& =\Sigma 1_{3} t_{2} \cdot\left[\left(R^{(1)} \cdot c\right) \cdot \omega\left(S^{-1}\left(1_{2} t_{1} R^{(2)}\right) 1_{1} h \cdot m\right)\right] \\
& =\Sigma t_{2} \cdot\left[\left(R^{(1)} \cdot c\right) \cdot \omega\left(S^{-1}\left(1_{2} t_{1} R^{(2)}\right) 1_{1} h \cdot m\right)\right] \\
& =\Sigma t_{2} \cdot\left[\left(R^{(1)} \cdot c\right) \cdot \omega\left(S^{-1}\left(t_{1} R^{(2)}\right) h \cdot m\right)\right]=\widehat{\omega}(h \cdot m),
\end{aligned}
$$

on the other hand, since $\Sigma h_{2} S^{-1}\left(h_{1}\right)=\bar{\Pi}^{L}(h)$ and

$$
\Sigma \bar{\Pi}^{L}\left(h_{1}\right) \otimes h_{2}=\Sigma 1_{1} \otimes 1_{2} h,
$$

for $h \in H$ also by [18], we have

$$
\begin{array}{rll}
\widehat{\omega}(a \cdot m) & = & \Sigma t_{2} \cdot\left[\left(R^{(1)} \cdot c\right) \cdot \omega\left(S^{-1}\left(t_{1} R^{(2)}\right) \cdot(a \cdot m)\right)\right] \\
& \stackrel{(12)}{=} & \Sigma t_{3} \cdot\left[\left(R^{(1)} \cdot c\right) \cdot \omega\left(\left(S^{-1}\left(t_{2} R_{2}^{(2)}\right) \cdot a\right) \cdot\left(S^{-1}\left(t_{1} R_{1}^{(2)}\right) \cdot m\right)\right)\right] \\
& \stackrel{(8)}{=} & \Sigma t_{3} \cdot\left[\left(R^{(1)} r^{(1)} \cdot c\right)\left(S^{-1}\left(t_{2} R^{(2)}\right) \cdot a\right) \cdot \omega\left(S^{-1}\left(t_{1} r^{(2)}\right) \cdot m\right)\right] \\
& = & \Sigma t_{3} \cdot\left[\left(R^{(1)} r^{(1)} \cdot c\right)\left(S^{-1}\left(R^{(2)}\right) S^{-1}\left(t_{2}\right) \cdot a\right) \cdot \omega\left(S^{-1}\left(t_{1} r^{(2)}\right) \cdot m\right)\right] \\
& \stackrel{(14)}{=} & \Sigma t_{3} \cdot\left[\left(S^{-1}\left(t_{2}\right) \cdot a\right)\left(R^{(1)} \cdot c\right) \cdot \omega\left(S^{-1}\left(t_{1} R^{(2)}\right) \cdot m\right)\right] \\
& = & \Sigma\left[t_{3} S^{-1}\left(t_{2}\right) \cdot a\right] \cdot\left[t_{4} \cdot\left(\left(R^{(1)} \cdot c\right) \cdot \omega\left(S^{-1}\left(t_{1} R^{(2)}\right) \cdot m\right)\right)\right] \\
& =\Sigma\left[\bar{\Pi}^{L}\left(t_{2}\right) \cdot a\right] \cdot\left[t_{3} \cdot\left(\left(R^{(1)} \cdot c\right) \cdot \omega\left(S^{-1}\left(t_{1} R^{(2)}\right) \cdot m\right)\right)\right] \\
& \stackrel{(21)}{=} & \Sigma\left[1_{1} \cdot a\right] \cdot\left[1_{2} t_{2} \cdot\left(\left(R^{(1)} \cdot c\right) \cdot \omega\left(S^{-1}\left(t_{1} R^{(2)}\right) \cdot m\right)\right)\right] \\
& = & \Sigma a \cdot\left[t_{2} \cdot\left(\left(R^{(1)} \cdot c\right) \cdot \omega\left(S^{-1}\left(t_{1} R^{(2)}\right) \cdot m\right)\right)\right]=a \cdot \widehat{\omega}(m) .
\end{array}
$$

Hence, $\widehat{\omega}$ is an $A \# H$-module morphism.

Now, we assume that $P$ is a left $A \# H$-module, which is projective as an $A$ module. What we will do next is to show that $P$ is projective as an $A \# H$-module as well. Let $\alpha: M \rightarrow N$ be an $A \# H$-epimorphism and $\beta: P \rightarrow N$ be an $A \# H$ module morphism. Then, there exists an $A$-module morphism $\omega: P \rightarrow M$ such that $\alpha \circ \omega=\beta$ since $P$ is a projective $A$-module. Take the map $\widehat{\omega}: P \rightarrow M$ as in (19). We claim that $\alpha \circ \widehat{\omega}=\beta$, which follows that $P$ is a projective $A \# H$-module.

Indeed, by Proposition 5.6 in [12], we have

$$
\Sigma \Pi^{L}\left(R^{(1)}\right) \otimes R^{(2)}=\Sigma 1_{2} \otimes 1_{1},
$$


then for any $p \in P$, since $\alpha$ is $A \# H$-linear and $c$ is a trace one element, we obtain

$$
\begin{aligned}
& \alpha \circ \widehat{\omega}(p)=\alpha\left(\Sigma t_{2} \cdot\left(\left(R^{(1)} \cdot c\right) \cdot\left(\omega\left(S^{-1}\left(t_{1} R^{(2)}\right) \cdot p\right)\right)\right)\right)=\Sigma t_{2} \cdot\left[\left(R^{(1)} \cdot c\right) \cdot \alpha \circ \omega\left(S^{-1}\left(t_{1} R^{(2)}\right) \cdot p\right)\right] \\
& =\Sigma\left[t_{2} R^{(1)} \cdot c\right] \cdot\left[t_{3} \cdot \beta\left(S^{-1}\left(t_{1} R^{(2)}\right) \cdot p\right)\right]=\Sigma\left[t_{2} R^{(1)} \cdot c\right] \cdot \beta\left(t_{3} S^{-1}\left(t_{1} R^{(2)}\right) \cdot p\right) \\
& \stackrel{(6)}{=} \quad \Sigma\left[R^{(1)} t_{1} \cdot c\right] \cdot \beta\left(S^{-1}\left(R^{(2)} t_{2} S\left(t_{3}\right)\right) \cdot p\right)=\Sigma\left[R^{(1)} t_{1} \cdot c\right] \cdot \beta\left(S^{-1}\left(R^{(2)} \Pi^{L}\left(t_{2}\right)\right) \cdot p\right) \\
& \stackrel{(20)}{=} \Sigma\left[R^{(1)} 1_{1} t \cdot c\right] \cdot \beta\left(S^{-1}\left(R^{(2)} 1_{2}\right) \cdot p\right)=\Sigma\left[R^{(1)} \cdot 1_{A}\right] \cdot \beta\left(S^{-1}\left(R^{(2)}\right) \cdot p\right) \\
& =\Sigma\left[\Pi^{L}\left(R^{(1)}\right) \cdot 1_{A}\right] \cdot \beta\left(S^{-1}\left(R^{(2)}\right) \cdot p\right) \stackrel{(22)}{=} \Sigma\left[1_{2} \cdot 1_{A}\right] \cdot \beta\left(S^{-1}\left(1_{1}\right) \cdot p\right) \\
& =\Sigma\left[\Pi^{L}\left(1_{1}\right) \cdot 1_{A}\right] \cdot\left[1_{2} \cdot \beta(p)\right]=\Sigma\left[1_{1} \cdot 1_{A}\right] \cdot\left[1_{2} \cdot \beta(p)\right]=\beta(p) \text {. }
\end{aligned}
$$

Hence, we complete the proof of Lemma 2.1.

Corollary 2.2. If $H$ is semisimple and a left $A \# H$-module $M$ is projective as an $A$-module, then $M$ is also projective as an A\#H-module.

Proof. Since $H$ is semisimple, there is a left integral $t \in H$ such that $\Pi^{L}(t)=1_{H}$ by [3]. Then $t \cdot 1_{A}=\Pi^{L}(t) \cdot 1_{A}=1_{A}$. If choose $c=1_{A}$ as the trace one element, then we have that $M$ is a projective $A \# H$-module by Lemma 2.1.

Let $B / C$ be a weak $H$-Galois extension. We use following formal notation for the inverse of can: for any $h \in H$,

$$
\operatorname{can}^{-1}\left(1_{B} \otimes_{H^{R}} h\right)=\Sigma l_{i}(h) \otimes r_{i}(h) \in B \otimes_{C} B,
$$

such that

$$
\Sigma l_{i}(h) r_{i}(h)_{(0)} \otimes_{H^{R}} r_{i}(h)_{(1)}=1_{B} \otimes_{H^{R}} h,
$$

by definition.

Lemma 2.3. Let $H$ be a finite dimensional weak Hopf algebra with a dual pair of left integrals $(T, t)$, and $B / C$ a weak right $H$-Galois extension. Define $\operatorname{tr}(a)=$ $a_{(0)} T\left(a_{(1)}\right)$ for all $a \in B$. Then, $t r$ is a $C$-bimodule map from $B$ onto $C$, and the following formula holds:

$$
\Sigma l_{i}\left(S^{-1}(t)\right) \operatorname{tr}\left(r_{i}\left(S^{-1}(t)\right)\right)=1_{B}=\Sigma \operatorname{tr}\left(l_{i}\left(S^{-1}(t)\right)\right) r_{i}\left(S^{-1}(t)\right) .
$$

Proof. Since $T \in H^{*}$ is a left integral, by [13] we have $\Sigma g_{1}\left\langle T, h g_{2}\right\rangle=\Sigma S\left(h_{1}\right)\left\langle T, h_{2} g\right\rangle$ for all $h, g \in H$. Then, for any $a \in B$,

$$
\begin{aligned}
\Sigma \rho\left(a_{(0)} T\left(a_{(1)}\right)\right) & =\Sigma a_{(0)} \otimes a_{(1)} T\left(a_{(2)}\right) \\
& =\Sigma a_{(0)} \otimes S\left(1_{1}\right)\left\langle T, 1_{2} a_{(1)}\right\rangle \in B \otimes H^{L},
\end{aligned}
$$

so $\operatorname{tr}(B) \subseteq C$. 
For all $a \in B, x \in C$, since

$$
\begin{aligned}
\operatorname{tr}(a x) & =\Sigma a_{(0)} x_{(0)} T\left(a_{(1)} x_{(1)}\right)=\Sigma a_{(0)} 1_{(0)} x T\left(a_{(1)} 1_{(1)}\right) \\
& =\Sigma a_{(0)} x T\left(a_{(1)}\right)=\operatorname{tr}(a) x, \\
\operatorname{tr}(x a) & =\Sigma x_{(0)} a_{(0)} T\left(x_{(1)} a_{(1)}\right)=\Sigma x 1_{(0)} a_{(0)} T\left(1_{(1)} a_{(1)}\right) \\
& =\Sigma x a_{(0)} T\left(a_{(1)}\right)=x \operatorname{tr}(a),
\end{aligned}
$$

$\operatorname{tr}$ is a $C$-bimodule map.

Furthermore, since the following diagrams
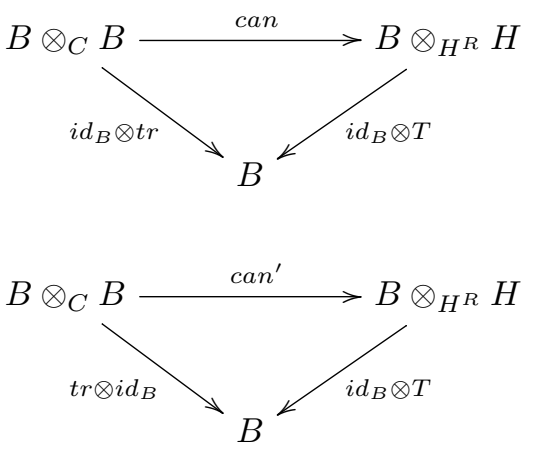

commute, and $T\left(S^{-1}(t)\right)=1_{k}$ by the proof of Theorem 6.4 in [17], we have

$$
\begin{array}{ll} 
& \left(i d_{B} \otimes T\right) \circ \operatorname{can}=i d_{B} \otimes_{C} \operatorname{tr} \\
\Longrightarrow \quad & \left(i d_{B} \otimes T\right) \circ \operatorname{can}\left(\Sigma l_{i}\left(S^{-1}(t)\right) \otimes_{C} r_{i}\left(S^{-1}(t)\right)\right)=\left(i d_{B} \otimes_{C} \operatorname{tr}\right)\left(\Sigma l_{i}\left(S^{-1}(t)\right) \otimes_{C} r_{i}\left(S^{-1}(t)\right)\right) \\
\Longrightarrow \quad & 1_{B} T\left(S^{-1}(t)\right)=\Sigma l_{i}\left(S^{-1}(t)\right) \operatorname{tr}\left(r_{i}\left(S^{-1}(t)\right)\right) \\
\Longrightarrow & 1_{B}=\Sigma l_{i}\left(S^{-1}(t)\right) \operatorname{tr}\left(r_{i}\left(S^{-1}(t)\right)\right),
\end{array}
$$

and on the other hand,

$$
\begin{array}{ll} 
& \left(i d_{B} \otimes T\right) \circ \operatorname{can}^{\prime}=\operatorname{tr} \otimes_{C} i d_{B} \\
\Longrightarrow \quad & \left(i d_{B} \otimes T\right) \circ \psi \circ \operatorname{can}\left(\Sigma l_{i}\left(S^{-1}(t)\right) \otimes_{C} r_{i}\left(S^{-1}(t)\right)\right)=\left(\operatorname{tr} \otimes_{C} i d_{B}\right)\left(\Sigma l_{i}\left(S^{-1}(t)\right) \otimes_{C} r_{i}\left(S^{-1}(t)\right)\right) \\
\Longrightarrow \quad & \left(i d_{B} \otimes T\right) \circ \psi\left(1_{B} \otimes_{H^{R}} S^{-1}(t)\right)=\Sigma \operatorname{tr}\left(l_{i}\left(S^{-1}(t)\right)\right) r_{i}\left(S^{-1}(t)\right) \\
\Longrightarrow \quad\left(i d_{B} \otimes T\right)\left(\Sigma 1_{(0)} \otimes_{H^{R}} 1_{(1)} t\right)=\Sigma \operatorname{tr}\left(l_{i}\left(S^{-1}(t)\right)\right) r_{i}\left(S^{-1}(t)\right) \\
\Longrightarrow \quad\left(i d_{B} \otimes T\right)\left(1_{B} \otimes_{H} t\right)=\Sigma \operatorname{tr}\left(l_{i}\left(S^{-1}(t)\right)\right) r_{i}\left(S^{-1}(t)\right) \\
\Longrightarrow \quad 1_{B} T(t)=\Sigma \operatorname{tr}\left(l_{i}\left(S^{-1}(t)\right)\right) r_{i}\left(S^{-1}(t)\right) \\
\Longrightarrow \quad 1_{B}=\Sigma \operatorname{tr}\left(l_{i}\left(S^{-1}(t)\right)\right) r_{i}\left(S^{-1}(t)\right) .
\end{array}
$$

The following proposition extends Theorem 5 in [5].

Proposition 2.4. Let $H$ be a finite dimensional weak Hopf algebra with a dual pair of left integrals $(T, t)$, and $B / C$ a weak right $H$-Galois extension. Let $V$ be a left $B$-module and $W$ a left $C$-module. Then there exists a space isomorphism $\gamma:{ }_{C} \operatorname{Hom}(V, W) \rightarrow{ }_{B} H o m\left(V, B \otimes_{C} W\right), \gamma(f)(v)=\Sigma l_{i}\left(S^{-1}(t)\right) \otimes_{C} f\left(r_{i}\left(S^{-1}(t)\right) v\right)$. 
In particular, the map $\gamma:{ }_{C} \operatorname{Hom}(B, W) \rightarrow{ }_{B} \operatorname{Hom}\left(B, B \otimes_{C} W\right) \cong B \otimes_{C} W$, is left B-linear isomorphic, where the B-module structures of ${ }_{C} H o m(B, W)$ and ${ }_{B} H \operatorname{Hom}\left(B, B \otimes_{C} W\right)$ are induced by the right multiplication of $B$, respectively.

Proof. For any $b \in B$, we have

$$
\Sigma b l_{i}\left(S^{-1}(t)\right) \otimes_{C} r_{i}\left(S^{-1}(t)\right)=\Sigma l_{i}\left(S^{-1}(t)\right) \otimes_{C} r_{i}\left(S^{-1}(t)\right) b
$$

Indeed, since $S^{-1}(t)$ is the right integral of $H$ and $\Sigma b_{(0)} \otimes \Pi^{R}\left(b_{(1)}\right)=\Sigma b 1_{(0)} \otimes$ $\Pi^{R}\left(1_{(1)}\right)$ for all $b \in B$ (cf. [19]), applying can to the both sides of above equation, we obtain

$$
\begin{aligned}
\operatorname{can}\left(\Sigma b l_{i}\left(S^{-1}(t)\right) \otimes_{C} r_{i}\left(S^{-1}(t)\right)\right) & =\Sigma b l_{i}\left(S^{-1}(t)\right) r_{i}\left(S^{-1}(t)\right)_{(0)} \otimes_{H^{R}} r_{i}\left(S^{-1}(t)\right)_{(1)} \\
& =b \otimes_{H^{R}} S^{-1}(t) \\
\operatorname{can}\left(\Sigma l_{i}\left(S^{-1}(t)\right) \otimes_{C} r_{i}\left(S^{-1}(t)\right) b\right) & =\Sigma l_{i}\left(S^{-1}(t)\right) r_{i}\left(S^{-1}(t)\right)_{(0)} b_{(0)} \otimes_{H^{R}} r_{i}\left(S^{-1}(t)\right)_{(1)} b_{(1)} \\
& =\Sigma b_{(0)} \otimes_{H^{R}} S^{-1}(t) b_{(1)}=\Sigma b_{(0)} \otimes_{H^{R}} S^{-1}(t) \Pi^{R}\left(b_{(1)}\right) \\
& =\Sigma b 1_{(0)} \otimes_{H^{R}} S^{-1}(t) \Pi^{R}\left(1_{(1)}\right)=\Sigma b 1_{(0)} \otimes_{H^{R}} S^{-1}(t) 1_{(1)} \\
& =\left(b \otimes_{H^{R}} S^{-1}(t)\right)\left(\Sigma 1_{(0)} \otimes_{H^{R}} 1_{(1)}\right) \\
& =b \otimes_{H^{R}} S^{-1}(t) .
\end{aligned}
$$

Next, we show that the map $\gamma$ is well-defined. For any $b \in B, v \in V$ and $f \in{ }_{C} H o m(V, W)$,

$$
\begin{aligned}
\gamma(f)(b v) & =\Sigma l_{i}\left(S^{-1}(t)\right) \otimes_{C} f\left(r_{i}\left(S^{-1}(t)\right) b v\right) \\
\stackrel{(24)}{=} & \Sigma b l_{i}\left(S^{-1}(t)\right) \otimes_{C} f\left(r_{i}\left(S^{-1}(t)\right) v\right) \\
= & b \gamma(f)(v) .
\end{aligned}
$$

Hence, the map $\gamma(f)$ is left $B$-linear.

For any $g \in{ }_{B} \operatorname{Hom}\left(V, B \otimes_{C} W\right)$, define

$$
\eta:{ }_{B} \operatorname{Hom}\left(V, B \otimes_{C} W\right) \rightarrow{ }_{C} H o m(V, W), \eta(g)=\left(\operatorname{tr} \otimes_{C} i d_{W}\right) \circ g .
$$

It is obvious that $\eta(g)$ are left $C$-linear because of the $C$-linearity of $t r$ and $g$.

Now by Lemma 2.3, we have for all $v \in V$,

$$
\begin{aligned}
\eta(\gamma(f))(v) & =\left(\operatorname{tr} \otimes_{C} i d_{W}\right)\left(\Sigma l_{i}\left(S^{-1}(t)\right) \otimes_{C} f\left(r_{i}\left(S^{-1}(t)\right) v\right)\right) \\
& =\Sigma \operatorname{tr}\left(l_{i}\left(S^{-1}(t)\right)\right) f\left(r_{i}\left(S^{-1}(t)\right) v\right) \\
& =f\left(\Sigma t r\left(l_{i}\left(S^{-1}(t)\right)\right) r_{i}\left(S^{-1}(t)\right) v\right) \\
& =f(v) .
\end{aligned}
$$


On the other hand, let $v \in V$ and denote $g(v)=\Sigma b_{i} \otimes_{C} w_{j}$, then for all $b \in B$ we have $g(b v)=\Sigma b b_{j} \otimes_{C} w_{j}$. It follows that

$$
\begin{aligned}
\gamma(\eta(g))(v) & =\Sigma l_{i}\left(S^{-1}(t)\right) \otimes_{C} \eta(g)\left(r_{i}\left(S^{-1}(t)\right) v\right) \\
& =\Sigma l_{i}\left(S^{-1}(t)\right) \otimes_{C}\left(\operatorname{tr} \otimes_{C} i d_{W}\right) \circ g\left(r_{i}\left(S^{-1}(t)\right) v\right) \\
& =\Sigma l_{i}\left(S^{-1}(t)\right) \otimes_{C} \operatorname{tr}\left(r_{i}\left(S^{-1}(t)\right) b_{j}\right) w_{j} \\
& =\Sigma l_{i}\left(S^{-1}(t)\right) \operatorname{tr}\left(r_{i}\left(S^{-1}(t)\right) b_{j}\right) \otimes_{C} w_{j} \\
& \stackrel{(24)}{=} \Sigma b_{j} l_{i}\left(S^{-1}(t)\right) \operatorname{tr}\left(r_{i}\left(S^{-1}(t)\right)\right) \otimes_{C} w_{j} \\
& =\Sigma b_{j} \otimes_{C} w_{j}=g(v) .
\end{aligned}
$$

This shows that $\eta \circ \gamma$ and $\gamma \circ \eta$ are identities, so that we have our desired isomorphism.

Lemma 2.5. For any left $A \# H$-module $M$, define a right $A$-action on $M$ by

$$
\varphi: M \otimes A \rightarrow M, m \otimes a \mapsto m<a:=\Sigma\left(R^{(1)} \cdot a\right) \cdot\left(S^{-1}\left(R^{(2)}\right) \cdot m\right) .
$$

for all $a \in A$ and $m \in M$. Then $M$ becomes an $(A, A)$-bimodule and an $\left(H^{L}, A\right)$ bimodule.

Proof. $M$ is a right $A$-module. For any $a, b \in A$ and $m \in M$, we have

$$
\begin{aligned}
& m<1_{A}=\Sigma\left(R^{(1)} \cdot 1_{A}\right) \cdot\left(S^{-1}\left(R^{(2)}\right) \cdot m\right)=\Sigma\left(\Pi^{L}\left(R^{(1)}\right) \cdot 1_{A}\right) \cdot\left(S^{-1}\left(R^{(2)}\right) \cdot m\right) \\
& \stackrel{(22)}{=} \Sigma\left(1_{2} \cdot 1_{A}\right) \cdot\left(S^{-1}\left(1_{1}\right) \cdot m\right)=\Sigma\left(S\left(1_{1}\right) \cdot 1_{A}\right) \cdot\left(1_{2} \cdot m\right) \\
& =\Sigma\left(1_{1} \cdot 1_{A}\right) \cdot\left(1_{2} \cdot m\right)=m, \\
& (m \angle a) \angle b=\Sigma\left(R^{(1)} \cdot b\right) \cdot\left(S^{-1}\left(R^{(2)}\right) \cdot(m \angle a)\right) \\
& =\Sigma\left(R^{(1)} \cdot b\right) \cdot\left(S^{-1}\left(R^{(2)}\right) \cdot\left(\left(r^{(1)} \cdot a\right) \cdot\left(S^{-1}\left(r^{(2)}\right) \cdot m\right)\right)\right) \\
& =\Sigma\left(R^{(1)} \cdot b\right)\left(S^{-1}\left(R_{2}^{(2)}\right) r^{(1)} \cdot a\right) \cdot\left(S^{-1}\left(r^{(2)} R_{1}^{(2)}\right) \cdot m\right) \\
& \stackrel{(8)}{=} \Sigma\left(R^{(1)} \breve{R}^{(1)} \cdot b\right)\left(S^{-1}\left(R^{(2)}\right) r^{(1)} \cdot a\right) \cdot\left(S^{-1}\left(r^{(2)} \breve{R}^{(2)}\right) \cdot m\right) \\
& \stackrel{(14)}{=} \quad \Sigma\left(r^{(1)} \cdot a\right)\left(\breve{R}^{(1)} \cdot b\right) \cdot\left(S^{-1}\left(r^{(2)} \breve{R}^{(2)}\right) \cdot m\right) \\
& \stackrel{(7)}{=} \quad \Sigma\left(R_{1}^{(1)} \cdot a\right)\left(R_{2}^{(1)} \cdot b\right) \cdot\left(S^{-1}\left(R^{(2)}\right) \cdot m\right) \\
& =\Sigma\left(R^{(1)} \cdot(a b)\right) \cdot\left(S^{-1}\left(R^{(2)}\right) \cdot m\right)=m\llcorner(a b) .
\end{aligned}
$$

What we next do is to prove that $\varphi$ indeed makes $M$ into an $(A, A)$-bimodule.

$$
\begin{aligned}
(a \cdot m) \leftarrow b & =\Sigma\left(R^{(1)} \cdot b\right) \cdot\left(S^{-1}\left(R^{(2)}\right) \cdot(a \cdot m)\right) \\
& =\Sigma\left(R^{(1)} \cdot b\right)\left(S^{-1}\left(R_{2}^{(2)}\right) \cdot a\right) \cdot\left(S^{-1}\left(R_{1}^{(2)}\right) \cdot m\right) \\
& \stackrel{(8)}{=} \Sigma\left(R^{(1)} r^{(1)} \cdot b\right)\left(S^{-1}\left(R^{(2)}\right) \cdot a\right) \cdot\left(S^{-1}\left(r^{(2)}\right) \cdot m\right) \\
& \stackrel{(14)}{=} \Sigma a\left(r^{(1)} \cdot b\right) \cdot\left(S^{-1}\left(r^{(2)}\right) \cdot m\right) \\
& =a \cdot(m \leftarrow b) .
\end{aligned}
$$

For any $a \in A, h \in H$ and $m \in M$, we have

$$
h \cdot(m<a)=\Sigma\left(h_{1} \cdot m\right)<\left(h_{2} \cdot a\right) .
$$


In fact, by (6) we have $R^{-1} \Delta^{o p}(h)=\Delta(h) R^{-1}$, that is,

$$
\begin{aligned}
\Sigma S\left(R^{(1)}\right) h_{2} \otimes R^{(2)} h_{1} & =\Sigma h_{1} S\left(R^{(1)}\right) \otimes h_{2} R^{(2)}, \\
\Sigma R^{(1)} h_{2} \otimes S^{-1}\left(R^{(2)}\right) h_{1} & =\Sigma h_{1} R^{(1)} \otimes h_{2} S^{-1}\left(R^{(2)}\right) .
\end{aligned}
$$

Hence

$$
\begin{aligned}
h \cdot(m \leftarrow a) & =\Sigma h \cdot\left[\left(R^{(1)} \cdot a\right) \cdot\left(S^{-1}\left(R^{(2)}\right) \cdot m\right)\right] \\
& =\Sigma\left(h_{1} R^{(1)} \cdot a\right) \cdot\left(h_{2} S^{-1}\left(R^{(2)}\right) \cdot m\right) \\
& =\Sigma\left(R^{(1)} h_{2} \cdot a\right) \cdot\left(S^{-1}\left(R^{(2)}\right) h_{1} \cdot m\right) \\
& =\Sigma\left(h_{1} \cdot m\right) \leftarrow\left(h_{2} \cdot a\right) .
\end{aligned}
$$

Now, for any $x \in H^{L}$, since $\Delta(x)=\Sigma 1_{1} x \otimes 1_{2}$, we compute

$$
\begin{aligned}
x \cdot(m<a) & =\Sigma\left(x_{1} \cdot m\right)<\left(x_{2} \cdot a\right) \\
& =\Sigma\left(1_{1} x \cdot m\right)<\left(1_{2} \cdot a\right) \\
& \stackrel{(29)}{=}(x \cdot m) \leftarrow a .
\end{aligned}
$$

It follows that $M$ is an $\left(H^{L}, A\right)$-bimodule.

Now, we are ready to give a necessary and sufficient condition of the global dimension of weak smash product $A \# H$ equals to that of its subalgebra $A$, which is quantum commutative as a weak $H$-module algebra, as the main result of this section. In the following, we denote $g l \cdot \operatorname{dim}(A)(\operatorname{proj} \operatorname{dim}(A))$ by the global dimension (projective dimension) of an algebra $A$, respectively.

Theorem 2.6. Let $(H, R)$ be a finite dimension quasitriangular weak Hopf algebra, and $A$ a quantum commutative weak left $H$-module algebra. Then gl.dim $(A \# H)=$ $n$ if and only if $\operatorname{gl.dim}(A)=n$ and there exists a trace one element $c \in A$.

Proof. " $\Longrightarrow$ "Suppose that $\operatorname{gl} \cdot \operatorname{dim}(A \# H)=n<\infty$. Let $M$ be an arbitrary left $A \# H$-module. There is an $A \# H$-epimorphism $(A \# H) \otimes_{A} M \rightarrow M$ given by $(a \# h) \otimes_{A} m \mapsto(a \# h) \cdot m$. In view of the fact that ${ }_{A} \operatorname{Hom}(A \# H, M) \cong(A \# H) \otimes_{A} M$ as $A \# H$-modules by Proposition 2.4 since the extension $A \# H / A$ is weak right $H$ Galois (see [14]), we have an epimorphism

$$
{ }_{A} \operatorname{Hom}(A \# H, M) \rightarrow M \rightarrow 0 .
$$

Let $m=p \cdot \operatorname{dim}_{A \# H} A$, then $m \leq n$. Applying the functor ${ }_{A \# H} H o m(A,-)$ to (27), we get a long exact sequence

$$
\cdots \rightarrow{ }_{A \# H} \operatorname{Ext}^{m}\left(A,{ }_{A} \operatorname{Hom}(A \# H, M)\right) \rightarrow{ }_{A \# H} \operatorname{Ext}^{m}(A, M) \rightarrow 0 .
$$

Since

${ }_{A \# H} \operatorname{Ext} t^{t}\left(A,{ }_{A} \operatorname{Hom}(A \# H, M)\right) \cong{ }_{A \# H} \operatorname{Ext}^{t}\left(A,(A \# H) \otimes_{A} M\right) \cong_{A} \operatorname{Ext}^{t}(A, M)$, 
${ }_{A} \operatorname{Ext}^{t}(A, M)=0$ for all $t>0$, and so $m=0$. Hence $A$ is projective as an $A \# H$ module. Then there exists some $c \in A$ such that $t \cdot c=1_{A}$ by Proposition 3 in [21], and we claim that the map $\xi: A \# H \rightarrow A, a \# h \mapsto a \cdot \Pi^{L}(h)$ is a split $A \# H$-epimorphism.

It is obvious that $\xi$ is both well-defined from the fact that $\Pi^{L}$ is a left $H^{L_{-}}$ linear map by [3], and a split epimorphism. Hence it suffices to show that $\xi$ is an $A \# H$-linearity.

In fact, since $\Delta(x)=\Sigma x 1_{1} \otimes 1_{2}$ for $x \in H^{L}$, and

$$
\Sigma \varepsilon\left(h_{2} g\right) h_{1}=h S^{-1} \Pi^{L}(g)
$$

for $h, g \in H$, we obtain

$$
\begin{aligned}
\xi[(a \# h)(b \# g)]=\xi\left[\Sigma a\left(h_{1} \cdot b\right) \# h_{2} g\right]=\Sigma\left[a\left(h_{1} \cdot b\right)\right] \cdot \Pi^{L}\left(h_{2} g\right) \\
\quad=\Sigma S^{-1} \Pi^{L}\left(h_{2} g\right) \cdot\left[a\left(h_{1} \cdot b\right)\right]=\Sigma\left[S^{-1}\left(\Pi^{L}\left(h_{2} g\right)_{2}\right) \cdot a\right]\left[S^{-1}\left(\Pi^{L}\left(h_{2} g\right)_{1}\right) h_{1} \cdot b\right] \\
\quad=\Sigma\left[S^{-1}\left(1_{2}\right) \cdot a\right]\left[S^{-1}\left(\Pi^{L}\left(h_{2} g\right) 1_{1}\right) h_{1} \cdot b\right]=\Sigma\left[1_{1} \cdot a\right]\left[1_{2} S^{-1}\left(\Pi^{L}\left(h_{2} g\right)\right) h_{1} \cdot b\right] \\
\quad=a\left[\Sigma \epsilon\left(1_{1} h_{2} g\right) S^{-1}\left(1_{2}\right) h_{1} \cdot b\right]=a\left[\Sigma \epsilon\left(1_{1} 1_{2}^{\prime} h_{2} g\right) S^{-1}\left(1_{2}\right) 1_{1}^{\prime} h_{1} \cdot b\right] \\
\quad=a\left[\Sigma \epsilon\left(1_{2} h_{2} g\right) S^{-1}\left(1_{3}\right) 1_{1} h_{1} \cdot b\right]=a\left[\Sigma S^{-1}\left(1_{2}\right) 1_{1} h S^{-1}\left(\Pi^{L}(g) \cdot b\right]\right. \\
\quad=a\left[h S^{-1}\left(\Pi^{L}(g) \cdot b\right]=(a \# h) \cdot\left[S^{-1}\left(\Pi^{L}(g) \cdot b\right]\right.\right. \\
\quad=(a \# h) \cdot\left[b \cdot \Pi^{L}(g)\right]=(a \# h) \cdot \xi(b \# g) .
\end{aligned}
$$

We claim that $A$ is an $(A, A)$-bimodule direct summand of $A \# H$. Indeed, $A$ and $A \# H$ are $(A, A)$-bimodules via left multiplication and the action $\varphi$ defined in (25). Since $A$ is $H$-quantum commutative, $a<b=a b$ for all $a, b \in A$. Furthermore, $\xi$ is an $(A, A)$-bimodule homomorphism. For any $a, b \in A$ and $h \in H$, by

$$
\begin{aligned}
\xi((a \# h)-b) & =\xi\left[\Sigma\left(R^{(1)} \cdot b\right)\left(S^{-1}\left(R^{(2)}\right) \cdot(a \# h)\right)\right] \\
& \left.=\Sigma \xi\left[\left(R^{(1)} \cdot b\right)\left(S^{-1}\left(R_{2}^{(2)}\right) \cdot a\right) \# S^{-1}\left(R_{1}^{(2)}\right) h\right)\right] \\
& =\Sigma\left[\left(R^{(1)} \cdot b\right)\left(S^{-1}\left(R_{2}^{(2)}\right) \cdot a\right)\right] \cdot \Pi^{L}\left(S^{-1}\left(R_{1}^{(2)}\right) h\right) \\
& =\Sigma \varepsilon\left(1_{1} S^{-1}\left(R_{1}^{(2)}\right) h\right)\left[\left(R^{(1)} \cdot b\right)\left(S^{-1}\left(R_{2}^{(2)}\right) \cdot a\right)\right] \cdot 1_{2} \\
& =\Sigma \varepsilon\left(\Pi^{R}\left(1_{1}\right) S^{-1}\left(R_{1}^{(2)}\right) h\right) S^{-1}\left(1_{2}\right) \cdot\left[\left(R^{(1)} \cdot b\right)\left(S^{-1}\left(R_{2}^{(2)}\right) \cdot a\right)\right] \\
& =\Sigma \varepsilon\left(S^{-1}\left(1_{1}\right) S^{-1}\left(R_{1}^{(2)}\right) h\right)\left(S^{-1}\left(1_{3}\right) R^{(1)} \cdot b\right)\left(S^{-1}\left(R_{2}^{(2)} 1_{2}\right) \cdot a\right) \\
& =\Sigma \varepsilon\left(S^{-1}\left(R_{1}^{(2)} 1_{1}\right) h\right)\left(S^{-1}\left(1_{2}^{\prime}\right) R^{(1)} \cdot b\right)\left(S^{-1}\left(R_{2}^{(2)} 1_{2} 1_{1}^{\prime}\right) \cdot a\right) \\
& =\Sigma \varepsilon\left(S^{-1}\left(R_{1}^{(2)}\right) h\right)\left(S^{-1}\left(1_{2}\right) R^{(1)} \cdot b\right)\left(S^{-1}\left(R_{2}^{(2)} 1_{1}\right) \cdot a\right) \\
& =\Sigma \varepsilon\left(S^{-1}\left(R_{1}^{(2)}\right) h\right)\left(R^{(1)} \cdot b\right)\left(S^{-1}\left(R_{2}^{(2)}\right) \cdot a\right) \\
& \stackrel{(27)}{=} \Sigma\left(R^{(1)} \cdot b\right)\left(S^{-1}\left(R^{(2)}\right) S^{-1} \Pi^{L}(h) \cdot a\right) \\
& =\left(S^{-1} \Pi^{L}(h) \cdot a\right) b=\left(a \cdot \Pi^{L}(h)\right) b=\xi(a \# h) \leftarrow b .
\end{aligned}
$$

Therefore, for any left $A$-module $N$, the map $\xi \otimes i d_{N}:(A \# H) \otimes_{A} N \rightarrow N$ is a split $A$-epimorphism (the left $A$-action of $(A \# H) \otimes_{A} N$ via the left component). 
Hence, $N$ is a direct summand of $(A \# H) \otimes_{A} N$. It follows that

$$
\operatorname{proj.dim}_{A}(N) \leq \text { proj.dim } A\left((A \# H) \otimes_{A} N\right) .
$$

We now consider the left $A \# H$-module $(A \# H) \otimes_{A} N$. Since its any projective $A \# H$-module resolution is also a projective resolution over $A$, we have

$$
\operatorname{proj.dim}_{A}\left((A \# H) \otimes_{A} N\right) \leq \text { proj.dim } \operatorname{di\# H}_{A}\left((A \# H) \otimes_{A} N\right) .
$$

Thus $\operatorname{gl} \cdot \operatorname{dim}(A) \leq \operatorname{gl} \cdot \operatorname{dim}(A \# H)<\infty$.

" $\Longleftarrow$ We assume that $g l \cdot \operatorname{dim}(A)=n<\infty$ and $t \cdot c=1_{A}$ for some $c \in A$. Let $M$ be any left $A \# H$-module. If $M$ is projective as an $A$-module, then $M$ is also projective as an $A \# H$ by Lemma 2.1. Let $N$ be an $A \# H$-module. Consider an exact sequence of $A \# H$-modules

$$
0 \rightarrow X \rightarrow P_{n-1} \rightarrow \cdots \rightarrow P_{0} \rightarrow N \rightarrow 0
$$

where ${ }_{A \# H} P_{i}(i=0, \ldots, n-1)$ is projective, we have ${ }_{A} X$ is projective since

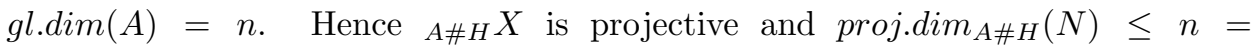
$\operatorname{proj.dim}_{A}(N)$. It follows that $\operatorname{gl} \operatorname{dim}(A \# H) \leq \operatorname{gl} \operatorname{dim}(A)$. The proof is completed.

As a consequent of Theorem 2.6 and Proposition 3 in [21], we have

Corollary 2.7. Let $(H, R)$ be a finite dimension quasitriangular weak Hopf algebra, and $A$ a quantum commutative weak left $H$-module algebra. Then

(1) $g l \cdot \operatorname{dim}(A \# H)=n$ if and only if $\operatorname{gl} \cdot \operatorname{dim}(A)=n$ and $A$ is a projective left A\#H-module.

(2) If $H$ is furthermore semisimple, then $\operatorname{gl} \operatorname{dim}(A \# H)=\operatorname{gl} \operatorname{dim}(A)$. Hence, $A$ is semisimple if and only if $A \# H$ is semisimple, and $A$ is hereditary if and only if $A \# H$ is hereditary.

Let us recall from [2] that the finitistic dimension of an algebra $A$ is defined by the formula

$$
\text { fin.dim }(A)=\sup \{\operatorname{proj} \cdot \operatorname{dim}(A)<\infty \mid M \text { is a left A-module and } \operatorname{proj} \cdot \operatorname{dim}(M)<\infty\} .
$$

In the following, we shall investigate the relation between the finitistic dimension of a quantum commutative weak $H$-module algebra $A$ and that of weak smash product $A \# H$. 
Proposition 2.8. Suppose that $(H, R)$ is a finite dimension quasitriangular weak Hopf algebra, its dual $H^{*}$ is semisimple, and let $A$ be a quantum commutative weak left $H$-module algebra. If there exists a trace one element $c \in A$, then fin.dim $(A \# H)=$ fin.dim $(A)$.

Proof. By Theorem 2.6, for any left $A \# H$-module $M$, we have proj.dim $\operatorname{d\# H}_{H}=$ proj.dim $_{A} M$. This implies that fin.dim $(A \# H) \leq \operatorname{fin} \cdot \operatorname{dim}(A)$.

Since $A \# H$ is a left $H^{*}$-module algebra in a natural way by [11], we can form a weak smash product $(A \# H) \# H^{*}$. Since $H^{*}$ is semisimple, $\operatorname{fin} \cdot \operatorname{dim}\left((A \# H) \# H^{*}\right) \leq$ fin.dim $(A \# H)$ by Lemma 6.2 in [9]. By Proposition 6.3 in [9], we know $(A \# H) \# H^{*}$ is Morita equivalent to $A$, then $\operatorname{fin} \cdot \operatorname{dim}\left((A \# H) \# H^{*}\right)=\operatorname{fin} \cdot \operatorname{dim}(A)$. Hence,

$$
\text { fin.dim }(A)=\operatorname{fin} \cdot \operatorname{dim}\left((A \# H) \# H^{*}\right) \leq \operatorname{fin} \cdot \operatorname{dim}(A \# H) \leq \operatorname{fin} \cdot \operatorname{dim}(A) .
$$

Therefore $\operatorname{fin} \cdot \operatorname{dim}(A \# H)=\operatorname{fin} \cdot \operatorname{dim}(A)$.

Corollary 2.9. Suppose that $(H, R)$ is a finite dimension semisimple quasitriangular weak Hopf algebra, its dual $H^{*}$ is semisimple, and let $A$ be a quantum commutative weak left $H$-module algebra. Then fin.dim $(A \# H)=f i n \cdot \operatorname{dim}(A)$.

\section{In the case of twisted weak Hopf algebra}

In this section, we always assume that the weak Hopf algebra $H$ is finite dimensional.

Definition 3.1. [13] A twist for $H$ is a pair $\left(\Theta, \Theta^{-1}\right)$, with

$$
\Theta \in \Delta\left(1_{H}\right)(H \otimes H), \Theta^{-1} \in(H \otimes H) \Delta\left(1_{H}\right), \text { and } \Theta \Theta^{-1}=\Delta\left(1_{H}\right),
$$

satisfying the following axioms:

$$
\begin{aligned}
(\varepsilon \otimes i d)(\Theta)=(i d \otimes \varepsilon)(\Theta) & =(\varepsilon \otimes i d)\left(\Theta^{-1}\right)=(i d \otimes \varepsilon)\left(\Theta^{-1}\right)=1_{H} \\
(\Delta \otimes i d)(\Theta)\left(\Theta \otimes 1_{H}\right) & =(i d \otimes \Delta)(\Theta)\left(\Theta \otimes 1_{H}\right) \\
\left(\Theta^{-1} \otimes 1_{H}\right)(\Delta \otimes i d)\left(\Theta^{-1}\right) & =\left(1_{H} \otimes \Theta^{-1}\right)(i d \otimes \Delta)\left(\Theta^{-1}\right) \\
(\Delta \otimes i d)\left(\Theta^{-1}\right)(i d \otimes \Delta)(\Theta) & =\left(\Theta \otimes 1_{H}\right)\left(1_{H} \otimes \Theta^{-1}\right) \\
(i d \otimes \Delta)\left(\Theta^{-1}\right)(\Delta \otimes i d)(\Theta) & =\left(1_{H} \otimes \Theta\right)\left(\Theta^{-1} \otimes 1_{H}\right)
\end{aligned}
$$

Lemma 3.2. Let $\left(\Theta, \Theta^{-1}\right)$ be a twist for $H$. Then there is a weak Hopf algebra $H_{\Theta}$ having the same algebra structure and counit as $H$ with a comultiplication and antipode given by

$$
\Delta_{\Theta}(h):=\Sigma h_{1}^{\Theta} \otimes h_{2}^{\Theta}=\Theta^{-1} \Delta(h) \Theta, S_{\Theta}(h)=v^{-1} S(h) v,
$$


for all $h \in H_{\Theta}$, where $v=\Sigma S\left(\Theta^{(1)}\right) \Theta^{(2)}$ is invertible with inverse $v^{-1}=\Sigma \Theta^{-(1)}$ $S\left(\Theta^{-(2)}\right)$ in $H_{\Theta}$. The counital maps of $H_{\Theta}$ are

$$
\Pi_{\Theta}^{L}(h)=\Sigma \varepsilon\left(\Theta^{(1)} h\right) \Theta^{(2)}, \Pi_{\Theta}^{R}(h)=\Sigma \varepsilon\left(h \Theta^{-(2)}\right) \Theta^{-(1)},
$$

for all $h \in H_{\Theta}$.

Proof. See Proposition 3.1.2 in [6] and Proposition 6.1.2 in [13].

Lemma 3.3. Let $\left(\Theta, \Theta^{-1}\right)$ be a twist for $H$, and $A$ a weak left $H$-module algebra. Define the operation $*$ on $A$ by $a * b=\Sigma\left(\Theta^{(1)} \cdot a\right)\left(\Theta^{(2)} \cdot b\right)$, Then $A$ becomes a weak left $H_{\Theta}$-module algebra, which we denote by $A_{\Theta}$.

Proof. First, $A_{\Theta}$ is an associative algebra with unit $1_{A}$. Since $\Sigma S\left(1_{1}\right) \otimes 1_{2}$ is a separability element of $H^{L}$ by [3], for any $x \in H^{L}$,

$$
\Sigma x S\left(1_{1}\right) \otimes 1_{2}=\Sigma S\left(1_{1}\right) \otimes 1_{2} x .
$$

Meanwhile, by $[6]$

$$
\begin{aligned}
\Sigma \Pi^{L}\left(\Theta^{(1)}\right) \Theta^{(2)} & =1_{H}, \\
\Sigma S^{-1} \Pi^{L}\left(\Theta^{(2)}\right) \Theta^{(1)} & =1_{H} .
\end{aligned}
$$

Then for any $a \in A_{\Theta}$, we have

$$
\begin{aligned}
1_{A} * a & =\Sigma\left(1_{1} \Theta^{(1)} \cdot 1_{A}\right)\left(1_{2} \Theta^{(2)} \cdot a\right)=\Sigma\left(1_{1} \Pi^{L}\left(\Theta^{(1)}\right) \cdot 1_{A}\right)\left(1_{2} \Theta^{(2)} \cdot a\right) \\
& =\Sigma\left(\Pi^{L}\left(\Theta^{(1)}\right) 1_{1} \cdot 1_{A}\right)\left(1_{2} \Theta^{(2)} \cdot a\right)=\Sigma\left(\Pi^{L}\left(\Theta^{(1)}\right) S\left(1_{1}\right) \cdot 1_{A}\right)\left(1_{2} \Theta^{(2)} \cdot a\right) \\
& \stackrel{(36)}{=} \Sigma\left(S\left(1_{1}\right) \cdot 1_{A}\right)\left(1_{2} \Pi^{L}\left(\Theta^{(1)}\right) \Theta^{(2)} \cdot a\right)=\Sigma\left(1_{1} \cdot 1_{A}\right)\left(1_{2} \cdot a\right) \stackrel{(37)}{=} a, \\
a * 1_{A} & \stackrel{=}{=} \Sigma\left(\Theta^{(1)} \cdot a\right)\left(\Theta^{(2)} \cdot 1_{A}\right)=\Sigma\left(1_{1} \Theta^{(1)} \cdot a\right)\left(1_{2} \Pi^{L}\left(\Theta^{(2)}\right) \cdot 1_{A}\right) \\
& \stackrel{(36)}{=} \Sigma\left(1_{1} S^{-1} \Pi^{L}\left(\Theta^{(2)}\right) \Theta^{(1)} \cdot a\right)\left(1_{2} \cdot 1_{A}\right)=\Sigma\left(1_{1} \cdot a\right)\left(1_{2} \cdot 1_{A}\right) \stackrel{(38)}{=} a .
\end{aligned}
$$

Hence, $1_{A}$ is the unit of $A_{\Theta}$. Moreover, for any $a, b, c \in A_{\Theta}$,

$$
\begin{aligned}
(a * b) * c & =\Sigma\left[\left(\Theta^{(1)} \cdot a\right)\left(\Theta^{(2)} \cdot b\right)\right] * c \\
& =\Sigma \bar{\Theta}^{(1)} \cdot\left[\left(\Theta^{(1)} \cdot a\right)\left(\Theta^{(2)} \cdot b\right)\right]\left(\bar{\Theta}^{(2)} \cdot c\right) \\
& =\Sigma\left(\bar{\Theta}_{1}^{(1)} \Theta^{(1)} \cdot a\right)\left(\bar{\Theta}_{2}^{(1)} \Theta^{(2)} \cdot b\right)\left(\bar{\Theta}^{(2)} \cdot c\right) \\
& \stackrel{(31)}{=} \Sigma\left(\bar{\Theta}^{(1)} \cdot a\right)\left(\bar{\Theta}_{1}^{(2)} \Theta^{(1)} \cdot b\right)\left(\bar{\Theta}_{2}^{(2)} \Theta^{(2)} \cdot c\right) \\
& =\Sigma\left(\bar{\Theta}^{(1)} \cdot a\right) \bar{\Theta}^{(2)} \cdot\left[\left(\Theta^{(1)} \cdot b\right)\left(\Theta^{(2)} \cdot c\right)\right]=a *(b * c) .
\end{aligned}
$$

That is $A_{\Theta}$ is associative, where $\Theta=\Sigma \Theta^{(1)} \otimes \Theta^{(2)}=\Sigma \bar{\Theta}^{(1)} \otimes \bar{\Theta}^{(2)}$. Hence, $A_{\Theta}$ is an associative algebra with unit $1_{A}$. Furthermore, it is obvious that $A_{\Theta}$ is an $H_{\Theta}$-module since $H_{\Theta}$ has the same algebra structure as $H$ by Lemma 3.2. 
Now, for any $a, b \in A_{\Theta}$ and $h \in H_{\Theta}$,

$$
\begin{aligned}
& h \cdot(a * b)=h \cdot\left[\Sigma\left(\Theta^{(1)} \cdot a\right)\left(\Theta^{(2)} \cdot b\right)\right]=\Sigma\left(h_{1} \Theta^{(1)} \cdot a\right)\left(h_{2} \Theta^{(2)} \cdot b\right) \\
& \quad=\Sigma\left(1_{1} h_{1} \Theta^{(1)} \cdot a\right)\left(1_{2} h_{2} \Theta^{(2)} \cdot b\right)=\Sigma\left(\bar{\Theta}^{(1)} \Theta^{-(1)} h_{1} \Theta^{(1)} \cdot a\right)\left(\bar{\Theta}^{(2)} \Theta^{-(2)} h_{2} \Theta^{(2)} \cdot b\right) \\
& \quad=\Sigma\left(\Theta^{-(1)} h_{1} \Theta^{(1)} \cdot a\right) *\left(\Theta^{-(2)} h_{2} \Theta^{(2)} \cdot b\right) \\
& \quad=\Sigma\left(h_{1}^{\Theta} \cdot a\right) *\left(h_{2}^{\Theta} \cdot b\right) .
\end{aligned}
$$

It follows that $A_{\Theta}$ is a weak left $H_{\Theta}$-module algebra.

We have the following which extends Theorem 1.7 in [7].

Proposition 3.4. Let $\left(\Theta, \Theta^{-1}\right)$ be a twist for $H$, and $A$ a weak left $H$-module algebra. Then left $A \# H$-module category $A \# H \mathfrak{M}$ and left $A_{\Theta} \# H_{\Theta}$-module category $A_{\Theta} \# H_{\Theta} \mathfrak{M}$ are equivalent.

Proof. Let $M$ be an $A \# H$-module. Define an $A_{\Theta} \# H_{\Theta}$-action by for all $a \in$ $A_{\Theta}, h \in H_{\Theta}$

$$
(a \# h) \rightarrow m=\left[\Sigma\left(\Theta^{(1)} \cdot a\right) \# \Theta^{(2)} h\right] \cdot m .
$$

Then, $M$ is an $A_{\Theta} \# H_{\Theta}$-module.

The above action (39) is well defined, since for any $a \in A_{\Theta}, h \in H_{\Theta}$ and $x \in H_{\Theta}^{L}$, and followed by the fact that $\Sigma S_{\Theta}\left(\Theta^{(1)}\right) \otimes \Theta^{(2)}$ is a separability element of $H_{\Theta}^{L}$, we have

$$
\begin{aligned}
(a \cdot x \# h) \rightarrow m & =\Sigma\left[\left(\Theta^{(1)} S_{\Theta}^{-1}(x) \cdot a\right) \# \Theta^{(2)} h\right] \cdot m \\
& =\Sigma\left[\left(\Theta^{(1)} \cdot a\right) \# \Theta^{(2)} x h\right] \cdot m \\
& =(a \# x h) \rightarrow m .
\end{aligned}
$$

Moreover, $\left(1_{A} \# 1_{H}\right) \rightarrow m=m$ for all $m \in M$.

$$
\begin{aligned}
\left(1_{A} \# 1_{H}\right) \rightarrow m & =\left[\Sigma\left(\Theta^{(1)} \cdot 1_{A}\right) \# \Theta^{(2)}\right] \cdot m \\
& =\left[\Sigma\left(\Pi^{L}\left(\Theta^{(1)}\right) \cdot 1_{A}\right) \# \Theta^{(2)}\right] \cdot m \\
& =\left[\Sigma\left(1_{A} \cdot \Pi^{L}\left(\Theta^{(1)}\right)\right) \# \Theta^{(2)}\right] \cdot m \\
& =\left[\Sigma 1_{A} \# \Pi^{L}\left(\Theta^{(1)}\right) \Theta^{(2)}\right] \cdot m \\
\stackrel{(37)}{=} & \left(1_{A} \# 1_{H}\right) \cdot m=m .
\end{aligned}
$$

At the same time, for any $a, b \in A_{\Theta}, h, g \in H_{\Theta}$, we have

$$
\begin{aligned}
{[(a \# h)(b \# g)] \rightarrow m=\left[\Sigma a *\left(h_{1}^{\Theta} \cdot b\right) \# h_{2}^{\Theta} g\right] \rightarrow m } \\
\quad=\left[\Sigma a *\left(\Theta^{-(1)} h_{1} \Theta^{(1)} \cdot b\right) \# \Theta^{-(2)} h_{2} \Theta^{(2)} g\right] \rightarrow m \\
=\left[\Sigma\left(\bar{\Theta}^{(1)} \cdot a\right)\left(\bar{\Theta}^{(2)} \Theta^{-(1)} h_{1} \Theta^{(1)} \cdot b\right) \# \Theta^{-(2)} h_{2} \Theta^{(2)} g\right] \rightarrow m \\
\quad \stackrel{(39)}{=}\left[\Sigma\left(\breve{\Theta}_{1}^{(1)} \bar{\Theta}^{(1)} \cdot a\right)\left(\breve{\Theta}_{2}^{(1)} \bar{\Theta}^{(2)} \Theta^{-(1)} h_{1} \Theta^{(1)} \cdot b\right) \# \breve{\Theta}^{(2)} \Theta^{-(2)} h_{2} \Theta^{(2)} g\right] \cdot m
\end{aligned}
$$




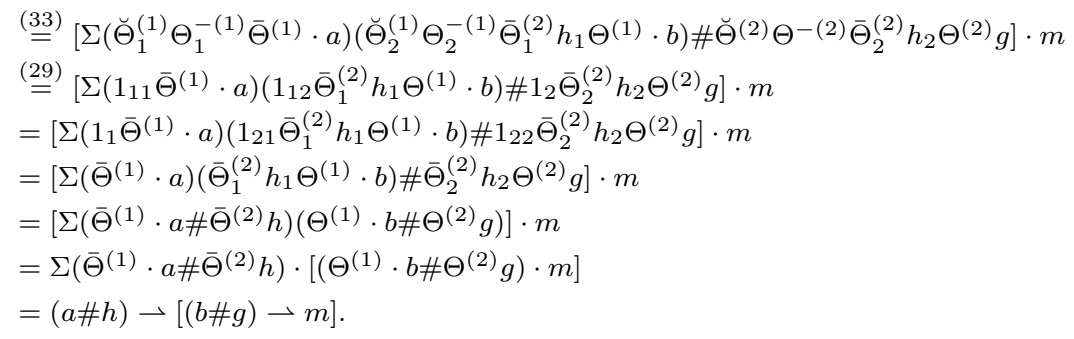

Hence, $M$ is a left $A_{\Theta} \# H_{\Theta}$-module.

Conversely, let $N$ be an $A_{\Theta} \# H_{\Theta}$-module. Define an $A \# H$-action by for all $a \in A, h \in H$

$$
(a \# h) \rightarrow n=\Sigma\left[\left(\Theta^{-(1)} \cdot a\right) \# \Theta^{-(2)} h\right] \cdot n .
$$

Then, $N$ is an $A \# H$-module.

The well-definition of the above action (40) can be similarly checked as before. For any $a, b \in A, h, g \in H$ and $n \in N$, it follows $\left(1_{A} \# 1_{H}\right) \rightarrow n=n$ from the equality $\Sigma \Pi^{L}\left(\Theta^{-(1)}\right) \Theta^{-(2)}=1_{H}$, and we have

$$
\begin{aligned}
(a \# h) \rightarrow[ & (b \# g) \rightarrow n]=(a \# h) \rightarrow\left[\Sigma\left(\Theta^{-(1)} \cdot b \# \Theta^{-(2)} g\right) \cdot n\right] \\
& =\Sigma\left(\Theta^{-(1)} \cdot a \# \Theta^{-(2)} h\right) \cdot\left(\breve{\Theta}^{-(1)} \cdot b \# \breve{\Theta}^{-(2)} g\right) \cdot n \\
& =\left[\Sigma\left(\Theta^{-(1)} \cdot a\right) *\left(\hat{\Theta}^{-(1)} \Theta_{1}^{-(2)} h_{1} \Theta^{(1)} \breve{\Theta}^{-(1)} \cdot b\right) \# \hat{\Theta}^{-(2)} \Theta_{2}^{-(2)} h_{2} \Theta^{(2)} \breve{\Theta}^{-(2)} g\right] \cdot n \\
& =\left[\Sigma\left(\Theta^{-(1)} \cdot a\right) *\left(\breve{\Theta}^{-(1)} \Theta_{1}^{-(2)} h_{1} \cdot b\right) \# \breve{\Theta}^{-(2)} \Theta_{2}^{-(2)} h_{2} g\right] \cdot n \\
& =\left[\Sigma\left(\Theta^{(1)} \Theta^{-(1)} \cdot a\right)\left(\Theta^{(2)} \breve{\Theta}^{-(1)} \Theta_{1}^{-(2)} h_{1} \cdot b\right) \# \breve{\Theta}^{-(2)} \Theta_{2}^{-(2)} h_{2} g\right] \cdot n \\
& \stackrel{(32)}{=}\left[\Sigma\left(\Theta^{(1)} \Theta^{-(1)} \breve{\Theta}_{1}^{-(1)} \cdot a\right)\left(\Theta^{(2)} \Theta^{-(2)} \breve{\Theta}_{2}^{-(1)} h_{1} \cdot b\right) \# \breve{\Theta}^{-(2)} h_{2} g\right] \cdot n \\
& =\left[\Sigma\left(\breve{\Theta}_{1}^{-(1)} \cdot a\right)\left(\breve{\Theta}_{2}^{-(1)} h_{1} \cdot b\right) \# \breve{\Theta}^{-(2)} h_{2} g\right] \cdot n \\
& =\left[\Sigma \breve{\Theta}^{-(1)} \cdot\left(a\left(h_{1} \cdot b\right)\right) \# \breve{\Theta}^{-(2)} h_{2} g\right] \cdot n \\
& =\left[\Sigma a\left(h_{1} \cdot b\right) \# h_{2} g\right] \rightarrow n \\
& =[(a \# h)(b \# g)] \rightarrow n .
\end{aligned}
$$

Hence, $N$ is a left $A \# H$-module.

Define the functor $\mathcal{F}:{ }_{A \# H} \mathfrak{M} \rightarrow{ }_{\Theta} \# H_{\Theta} \mathfrak{M}$ by $\mathcal{F}(M):=M$ as a $k$-space with the $A_{\Theta} \# H_{\Theta}$-module structure defined in (39), and the functor $\mathcal{G}:{ }_{A_{\Theta}} \# H_{\Theta} \mathfrak{M} \rightarrow{ }_{A \# H} \mathfrak{M}$ by $\mathcal{G}(N):=N$ as a $k$-space with the $A \# H$-module structure defined in (40). Then $(\mathcal{F}, \mathcal{G})$ are equivalent functors. Hence, the categories $A \# H \mathfrak{M}$ and $A_{\Theta} \# H_{\Theta} \mathfrak{M}$ are equivalent.

Since a twisting of the weak quasitriangular Hopf algebra $(H, R)$ is again quasitriangular with the structure given by $\left(H_{\Theta}, \Theta_{21}^{-1} R \Theta\right)$ (see Remark 6.1.3 in [13]), we have the following.

Theorem 3.5. Let $(H, R)$ be a finite dimension quasitriangular weak Hopf algebra with a twist $\left(\Theta, \Theta^{-1}\right)$, and $A$ a quantum commutative weak left $H$-module algebra. Then the following assertions are equivalent: 
(1) $\operatorname{gl} \cdot \operatorname{dim}(A \# H)=n$.

(2) $\operatorname{gl} \cdot \operatorname{dim}\left(A_{\Theta} \# H_{\Theta}\right)=n$.

(3) $\operatorname{gl} \operatorname{dim}(A)=n$ and $t \cdot c=1_{A}$ for some $c \in A$.

(4) $\operatorname{gl.dim}\left(A_{\Theta}\right)=n$ and $t \cdot c=1_{A}$ for some $c \in A$.

Proof. (1) and (3) are equivalent followed by Theorem 2.6. Since $A \# H$ and $A_{\Theta} \# H_{\Theta}$ are Morita equivalent by Proposition 3.4, gl.dim $(A \# H)=g l \cdot \operatorname{dim}\left(A_{\Theta} \# H_{\Theta}\right)$. Now to show (2) and (4) are equivalent, it suffices to show that if $A$ is quantum $H$-commutative, then $A_{\Theta}$ is quantum $H_{\Theta}$-commutative. That is to show that

$$
a * b=\Sigma\left(\Theta^{-(1)} R^{(2)} \Theta^{(2)} \cdot b\right) *\left(\Theta^{-(2)} R^{(1)} \Theta^{(1)} \cdot a\right)
$$

for all $a, b \in A_{\Theta}$, which can be easily checked.

Acknowledgment. The author would like to thank the referee for the valuable suggestions and comments.

\section{References}

[1] M. Auslander, I. Reiten and S. O. Smalø, Representation Theory of Artin Algebras, Cambridge University Press, Cambridge, 1995.

[2] H. Bass, Finitistic dimensions and a homological generalization of semiprimary rings, Trans. Amer. Math. Soc., 95 (1960), 466-488.

[3] G. Böhm, F. Nill and K. Szlachányi, Weak Hopf algebras (I): Integral theory and $C^{*}$-structure, J. Algebra, 221 (1999), 385-438.

[4] S. Caenepeel and E. De Groot, Modules over weak entwining structures, Contemp. Math., 267 (2000), 31-54.

[5] Y. Doi, Hopf extensions of algebras and Maschke type theorems, Israel J. Math., 72 (1990), 99-108.

[6] P. Etingof and D. Nikshych, Dynamical quantum groups at roots of 1, Duke Math. J., 108 (2001), 135-168.

[7] A. Giaquinto and J. J. Zhang, Bialgebra actions, twists, and universal deformation formulas, J. Pure Appl. Algebra, 128 (1998), 133-151.

[8] L. Jia and F. Li, Global dimension of weak smash product, J. Zhejiang Univ. Science A, 7 (2006), 2088-2092.

[9] L. Liu, B. L. Shen and S. H. Wang, On weak crossed products of weak Hopf algebras, Algebr. Represent. Theory, DOI 10.1007/s10468-011-9323-1, 16 (2013), 633-657. 
[10] S. Montgomery, Hopf Algebras and Their Actions on Rings, CBMS 82, Amer. Math. Soc. Providence, RI, 1993.

[11] D. Nikshych, A duality theorem for quantum groupoids, Contemp. Math., 267 (2000), 237-243.

[12] D. Nikshych, V. Turaev and L. Vainerman, Invariants of knots and 3-manifolds from quantum groupoids, Topo. Appl., 127 (2003), 91-123.

[13] D. Nikshych and L. Vainerman, Finite quantum groupoids and their applications, Math. Sci. Res. Inst. Publ., 43 (2002), 211-262.

[14] R. F. Niu, Y. Wang and L. Y. Zhang, The structure theorem of endomorphism algebras for weak Doi-Hopf modules, Acta Math. Hungar., 127 (2010), 273-290.

[15] A. B. R. Raposo, Crossed products for weak Hopf algebras, Comm. Algebra, 37 (2009), 2274-2289.

[16] M. E. Sweedler, Hopf Algebras, Mathematics Lecture Note Series, W. A. Benjamin, Inc., New York, 1969.

[17] P. Vecsernyés, Larson-Sweedler theorem and the role of grouplike elements in weak Hopf algebras, J. Algebra, 270 (2003), 471-520.

[18] Z. W. Wang, Y. Y. Chen and L. Y. Zhang, Total integrals for weak DoiKoppinen data, Algebr. Represent. Theory, 16 (2013), 931-953.

[19] Y. Wang and L. Zhang, The structure theorem and duality theorem for endomorphism algebras of weak Hopf algebras, J. Pure Appl. Algebra, 215 (2011), 1133-1145.

[20] S. Yang, Global dimension for Hopf actions, Comm. Algebra, 30 (2002), 36533667.

[21] L. Zhang and Y. Li, Homomorphisms, separable extensions and Morita maps for weak module algebras, Sib. Math. J., 52 (2011), 167-177.

[22] X. Y. Zhou, Homological dimension of weak Hopf-Galois extensions, Acta Math. Hungar., 138 (2013), 140-146.

\section{Zhong-wei Wang}

Department of Basic Sciences

Jinling Institute of Technology

Nanjing 211169, P.R. China

e-mail: wangzhongwei@jit.edu.cn 